\title{
Ueber die Plasminsäure.
}

Von

\author{
Alberto Ascoli.
}

(Aus dem physiologischen Institut in Marburg.)

(Der Redaction zugegangen am 2. August 1899.)

Diejenigen phosphorhaltigen Stoffe, welche man ursprünglich unter der Bezeichnung «Nuclein» zusammenfasste, haben sich als durchaus verschiedenartig erwiesen. Nachdem die Gruppe der «Paranucleinstoffe» abgetrennt und die Nucleine als Verbindungen von organischen phosphorhaltigen Säuren (Nucleinsäuren) mit Eiweisskörpern charakterisirt waren, ergab sich, dass die als Nucleinsäuren bezeichneten Verbindungen durchaus verschiedenartig sind. Nach $A$. Kossel ${ }^{1}$ ) können dieselben in 3 Gruppen eingetheilt werden : 1) Thymonucleinsäuren, 2) Gruppe der Inosinsäure und Guanylsäure, 3) Plasminsäure. Die Thymonucleinsäuren sind unter sich verschiedenartig. Zum Beispiel zeichnet sich eine derselben dadurch aus, dass sie gelatinirende Lösungen bildet, ${ }^{2}$ ) während andere diese Eigenschaft nicht besitzen.

Unter dem Namen «Plasminsäure» wurde von A. Kos sel ${ }^{3}$ ) eine aus Hefe dargestellte Substanz beschrieben. Ich will die Ergebnisse seiner Untersuchungen wörtlich wiedergeben: "Die Plasminsäure unterscheidet sich von der Nucleinsäure schon

1) Liebreich's Encyklopädie, Bd. III.

2) A. Kossel, Weitere Beiträge zur Kenntniss der Nucleinsäure. Archiv für Anatomie und Physiologie. Physiologische Abtheilung 1894, S. 195.

3) Archiv für Anatomie und Physiologie. Physiologische Abtheilung 1893, S. 160 und folgende. 
durch ihre Löslichkeitsverhältnisse. Sie löst sich auch nach längerem Aufbewahren leicht in Wasser, ebenso in sehr verdünnter, wässeriger Salzsäure und kann auf diese Weise von der Nucleinsäure getrennt werden. Sie fällt Eiweiss wie die Nucleinsäure. Ihre Analyse ergab, dass sie der Formel $\mathrm{C}_{15} \mathrm{H}_{28} \mathrm{~N}_{6} \mathrm{P}_{6} \mathrm{O}_{30}$ entspricht, mithin enthält sie doppelt soviel Phosphor wie die Nucleinsäure selbst. Unterwirft man sie der Spaltung durch siedende verdünnte Säuren, so gehen aus ihr die Nucleinbasen hervor, ferner entsteht eine noch nicht näher untersuchte stickstoffhaltige organische Substanz, welche ihren Stickstoff bei weiterer Einwirkung der Säuren als Ammoniak abgibt, und drittens bildet sich Phosphorsäure.

Neben der Plasminsäure und wahrscheinlich durch weitere Zersetzung aus ihr entsteht nun noch eine zweite Säure, über welche ich später berichten werde, aus deren Untersuchung hervorgeht, dass sie weniger Sauerstoff enthalten muss, als die Phosphorsäure. Wir haben hier wahrscheinlich eine Anhydridform der Phosphorsäure vor uns, und in der That entsprechen die Eigenschaften dieser Substanz im Wesentlichen denjenigen der Metaphosphorsäuren.»

Erwähnenswerth ist, dass L. Liebermann ${ }^{1}$ ) schon vorher aus der Hefe das Barytsalz einer Säure dargestellt hatte, die einige Eigenschaften mit der Metaphosphorsäure gemein haben sollte. L. Liebermann glaubt auch den Beweis geliefert zu haben, dass diese Säure Metaphosphorsäure ist. Indess halten die Analysen einer Kritik nicht Stand. ${ }^{2}$ ) Liebermann wurde bei seinen Untersuchungen von der Anschauung geleitet, dass die Nucleine nichts sind, als Verbindungen von Eiweiss mit Metaphosphorsäure, denen die metaphosphorsauren Salze des Xanthins und Guanins beigemischt sind. ${ }^{3}$ ) Die Unmöglichkeit dieser Anschauung liegt auf der Hand und ist genügend erwiesen. Völlig unabhängig von diesen Anschauungen ist die Frage, ob eine Anhydridform der Phosphorsäure als solche

1) Archiv für die gesammte Physiologie, Bd. XLVII, S. 155.

2) A. Kossel, loc. cit.

3) Centralblatt für die medic. Wissenschaften 1889, S. 210 u. 225. 
oder in organischer Bindung in den Organismen vorkommt. Die Untersuchungen über die Plasminsäure, welche ich auf Veranlassung von Prof. A. Kossel von Neuem aufgenommen habe, haben in der That neue Gründe für das Vorkommen der Metaphosphorsäure in den Organismen geliefert.

\section{Darstellung der Plasminsäure.}

Ich führte die Darstellung, von einigen Modificationen abgesehen, nach demselben Verfahren aus, welches von Prof. A. Kossel bei seinen früheren Untersuchungen benutzt worden war. Dasselbe ist folgendes:

Zwölf Liter untergährige gepresste Hefe wurden in drei Liter $30 \%$ iger Natronlauge gelöst, nach etwa einer Viertelstunde die Lösung mit zwei Liter Wasser verdünnt, mit zwei Liter 48\% iger Eisenchloridlösung gefällt und die Masse durch Spitzbeutel filtrirt. Das etwa vier bis fünf Liter betragende braune Filtrat wird in das gleiche Volumen einer Mischung von concentrirter Salzsäure und 85\% igem Alkohol unter Umrühren hineingegossen. Die Salzsäuremenge muss eben ausreichen, um die Flüssigkeit sauer zu machen, und wird jedesmal durch Titrirung des alkalischen Filtrats ermittelt. Man lässt den Niederschlag sich absetzen, hebert die darüber stehende Flüssigkeit $a b$, centrifugirt den Niederschlag, wäscht und trocknet ihn mit Alkohol und Aether und bringt ihn in das Vacuum. Die Ausbeute an diesem Rohprodukt (A) beträgt 40-80 g; sein Phosphorgehalt 5-10\%, im Mittel 7\%, A wird mit Wasser extrahirt, darauf filtrirt; das gelbe Filtrat von Neuem mit Salzsäure und Alkohol, dem etwas Aether zugesetzt wird, gefällt, der Niederschlag mit Alkohol und Aether gewaschen, getrocknet und ins Vacuum gebracht. Die Ausbeute an diesem zweiten Produkt (B) beträgt 5-10 g, sein Phosphorgehalt 13-23\%, gewöhnlich $14 \%$. Wird $B$ mit $0,1 \%$ iger wässeriger Salzsäure extrahirt, das opalisirende Filtrat durch Alkohol und etwas Aether gefällt, der Niederschlag mit Alkohol und Aether gewaschen und getrocknet, so erhält man die Plasminsäure; der in wässeriger Salzsäure ungelöst bleibende Theil, der auch ganz fehlen kann, ist Nucleinsäure. Die Ausbeute an Plasmin- 
säure beträgt etwa $4-5 \mathrm{~g}$ aus 12 Liter Hefe, in einzelnen Fällen mehr, aber auch weniger. Ihr Phosphorgehalt ist im Mittel $20 \%$, doch kommen Schwankungen bis $16 \%$ hinunter und 27\% hinauf mitunter vor. In einzelnen Fällen habe ich A direkt mit 1\% iger Salzsäure extrahirt und das Extract mit Alkohol und etwas Aether gefällt; der gewaschene und getrocknete Niederschlag stellte eine Säure von $16-18 \%$ Phosphor dar. In seltenen Fällen gelingt die Extraction von B mit $0,1 \%$ iger Salzsäure nicht, weil die Masse trübe durch das Filter läuft; in einem solchen Falle thut man gut, B wieder in Wasser zu lösen, mit Ammoniak zu neutralisiren und nun durch Alkohol und etwas Aether zu fällen; diese umgelöste Portion $\mathrm{B}$ ist nun wie gewöhnlich weiter $\mathrm{zu}$ behandeln.

\section{Eigenschaften der Plasminsäure.}

Das nach obiger Methode dargestellte Präparat von «Plasminsäure» stellt ein weisses oder graues Pulver dar, welches in Wasser sehr leicht löslich ist; Ammoniak ruft eine Gelbfärbung hervor. Eine concentrirte Lösung sieht gelblich aus und reagirt stark sauer. Sie gibt weder die Biuret- noch die Millon'sche Reaction und enthält keinen Schwefel. Bei der Zersetzung mittelst siedender Säuren gehen aus ihr die Nucleinbasen und Phosphorsäure hervor. Sie enthält circa 1\% Eisen; auf diesen Eisengehalt komme ich noch zurück. Von den Reactionen der wässerigen Lösung scheinen mir folgende besonders bemerkenswerth:

1) Sie fällt Eiweiss und Witte's Pepton bei saurer Reaction der Flüssigkeit.

2) Sie gibt mit Silbernitrat einen weissen Niederschlag, der leicht in Ammoniak, nur theilweise in Salpetersäure löslich ist.

3) Mit Chlorbaryurn gibt sie einen weissen flockigen Niederschlag, der unlöslich in Essigsäure, in Salzsäure leicht löslich ist.

4) Mit Eisenchlorid entsteht ein Niederschlag.

5) Nach Entfernung des Eisens mittels Schwefelammonium entsteht beim Kochen mit Phloroglucin und Salzsäure eine kirschrothe Färbung. 
6) Mit Schwefelsäure destillirt, entsteht Furfurol, durch Rothfärbung mit essigsaurem Anilin nachgewiesen.

7) Das Verhalten zu wässeriger Salzsäure ist je nach der Art der Darstellung und dem Phosphorgehalt ein verschiedenes. Ist die Extraction mit 0,1\% iger Salzsäure ausgeführt worden, so entsteht meist ein Niederschlag, ist hingegen 1\% ige Salzsäure benutzt worden, so bleibt die Flüssigkeit klar. Die phosphorreichsten Präparate geben keinen Niederschlag mit Salzsäure, wohl aber die phosphorärmeren.

Die letzteren Reactionen machen es wahrscheinlich, dass hier noch eine Mischung vorliegt, in der vermuthlich die eigentliche Plasminsäure enthalten ist. Ich habe zunächst die Frage nach der chemischen Natur der Plasminsäure selbst bei Seite gelassen und mich mit der phosphorreichen Substanz beschäftigt. Mit dem Worte Plasminsäure will ich im Folgenden lediglich das Produkt bezeichnen, welches nach obiger Darstellung gewonnen ist.

\section{Ist die phosphorreichere Substanz eine Metaphosphorsäure?}

Zur Unterscheidung der Metaphosphorsäure von der Orthophosphorsäure sind bisher vorwiegend folgende Reactionen benutzt worden:

1) die Eiweissfällung,

2) die Bildung des weissen Silbersalzes,

3) die Bildung unlöslicher Verbindungen mit primären Aminbasen, während secundäre und tertiäre Basen nicht gefällt werden. ${ }^{1}$ )

Diesen Reactionen möchte ich noch zwei hinzufügen, die ich in der Litteratur nicht vorgefunden habe und die beide auf dem Verhalten zu Eisenoxyd beruhen.

Wird eine Metaphosphorsäurelösung (aus Phosphorsäureanhydrid oder aus käuflicher Metaphosphorsäure dargestellt) vorsichtig mit Eisenchlorid versetzt, so ent-

1) Schlömann, Berichte der deutsch. chem. Gesellschaft, 1893, Jahrg. XXVI, S. 1020. 
steht Anfangs kein Niederschlag, oder es löst sich der entstehende im Ueberschuss der Säure; versetzt man nun die Lösung mit Ammoniak, so entsteht auch jetzt kein Niederschlag, sondern eine gelbe Färbung, die bei grösseren Mengen von Eisen portweinroth wird; eine analoge Reaction hat Rose ${ }^{1}$ ) für das Eisenoxydul beschrieben. Auch die Pyrophosphorsäure gibt diese Reaction, doch nicht die Ortophosphorsäure; auch habe ich sie bisher bei keiner anderen Mineralsäure gefunden. Die zweite Reaction beruht darauf, dass die Metaphosphorsäure das Eisenoxyd dem Nachweis als Berlinerblau bis zu einem gewissen Grade entzieht. Wird nämlich Eisenchlorid, wie oben beschrieben, mit Metaphosphorsäure in Lösung zusammengebracht, so entsteht, wenn nicht zuviel Eisenchlorid zugesetzt worden ist, bei Versetzen der Lösung mit Ferrocyankalium und Salzsäure höchstens eine Andeutung einer Grünfärbung, während dieselbe Menge Eisenchlorid ohne Metaphosphorsäurezusatz eine sehr kräftige Berlinerblaureaction zeigt. Dass die Metaphosphorsäure es ist, die das Eisen maskirt, geht auch daraus hervor, dass, wenn von einer solchen Lösung, die auf Ferrocyankalium und Salzsäure nicht reagirt, eine Probe mit Salzsäure gekocht wird, wodurch die Metaphosphorsäure in Orthophosphorsäure übergeführt wird, jetzt die früher vermisste Berlinerblaureaction in frappanter Weise eintritt. Für die Rhodanreaction gilt dasselbe.

Alle diese genannten Reactionen habe ich auch bei der «Plasminsäure» eintreten gesehen. Zur Schlömann'schen Reaction habe ich Phenylhydrazin als primäre Aminbase verwandt und sowohl mit freiem Phenylhydrazin in ätherischer als auch mit salzsaurem in wässeriger Lösung einen Niederschlag erhalten; als secundäre Aminbase wurde Piperidin, als tertiäre Pyridin verwandt, keines von beiden bewirkte einen Niederschlag. Die Maskirung des. Eisens habe ich, obgleich der Beweis hierfür schon dadurch gegeben war, dass das Eisen der Plasminsaure oft durch Ferrocyankalium und Salzsäure nicht nachweisbar ist, doch in schlagender Weise

1) H. Rose, Ueber die isomeren Modificationen der Phosphorsäure Pogg. 76, 1849.

Hoppe-Seyler's Zeitschrift f. physiol. Chemie. XXVIII. 
vor Augen führen wollen. Von der durch Extraction der trockenen gepulverten Plasminsäure mit Natronlauge vom Eisen grösstentheils befreiten Plasminsäure stellte ich eine nicht allzu verdünnte Lösung dar; zugleich bereitete ich mir eine verdünnte Eisenchloridlösung und theilte dieselbe in zwei gleiche Theile: der eine mit obiger Lösung von Plasminsäure zusammengebracht gab mit Ferrocyankalium und Salzsäure kaum eine Grünfärbung, der zweite, unter den identischen Bedingungen, aber ohne jenen Zusatz, gab mit Ferrocyankalium und Salzsäure die deutlichste Berlinerblaureaction.

Das weisse Silbersalz, das eisenhaltig ist, habe ich quantitativ analysirt, und bei einem Gehalte von $14,9 \%$ an Phosphor (für metaphosphorsaures Silber berechnet 16,57\% P) nur $1,7 \%$ Kohlenstoff und 0,7\% Wasserstoff nebst minimalen Mengen Stickstoff gefunden, so dass etwa auf 7 Atome Phosphor 2 Atome Kohlenstoff kamen. Es lag hier also eine sehr phosphorreiche organische Verbindung oder eine Mischung eines organischen Körpers mit einer anorganischen Phosphorverbindung vor.

Auch das Phenylhydrazinsalz habe ich zu quantitativen Bestimmungen herangezogen, und dabei folgende Mittelwerthe gefunden: $\mathrm{C}$ 36,1, $\mathrm{H} 4,9, \mathrm{~N} 15, \mathrm{P} 16$. Diese Verbindung ist jedenfalls noch kein reines Phenylhydrazinmetaphosphat, denn dieses verlangt: $\mathrm{C} 38,29, \mathrm{H} 4,78, \mathrm{~N} 14,89, \mathrm{P} 16,48$. Ich versuchte es zur weiteren Reinigung in das Silbersalz überzuführen, indem iç die alkalische Lösung des Phenylhydrazinsalzes mit Aether extrahirte und dann durch Fällung mit Silbernitrat das Silbersalz gewann, doch dieses enthielt noch Kohlenstoff, und zwar ergab sich auf 3 Atome Phosphor kaum 1 Atom Kohlenstoff. Es gelang mir sogar in diesem Silbersalz durch Zersetzung mit Schwefelammonium und Spaltung mittelst siedender Salzsäure Spuren von Nucleinbasen nachzuweisen.

Pohl') hat auf drei neue, aus dem Salze Graham's gewonnene Metaphosphate aufmerksam gemacht; nämlich auf das

1) J. Pohl, Bemerkungen über künstlich dargestellte Eiweissnucleine, Zeitschrift f. physiol. Chemie, Bd. XIII, S. 292. 
Guanidin-, das Strychnin- und das Chininsalz, von denen die beiden ersten krystallinisch sind, während das dritte sich als käsiger Niederschlng abscheidet. Auch die Lösung der nach obigem Verfahren dargestellten "Plasminsäure » gibt, nach Neutralisation mit Ammoniak, mit diesen Basen unlösliche Salze von genau demselben Verhalten, wie es Pohl für seine Metaphosphate angibt, und in der That ist es gelungen, auf diese Weise krystallisirte Verbindungen der in der "Plasminsäurelösung " enthaltenen phosphorreicheren Säure mit Guanidin und Strychnin darzustellen. Das Chininsalz ist, ebenso wie das aus dem Salze Grahams gewonnene, ein amorpher käsiger Niederschlag. Ich überzeugte mich durch Prüfung mit Silbernitrat, mit Magnesiamischung und durch die Eiweissfällung, dass in allen diesen Salzen die Gegenwart von Orthophosphaten ausgeschlossen war.

Das aus der Plasminsäure gewonnene Strychninsalz habe ich näher untersucht; es krystallisirt entweder in Nadeln oder in Prismen und rectangulären Tafeln; diese letzteren erscheinen bei krystallographischer Untersuchung schwach doppeltbrechend. Die Auslöschungsrichtung war parallel den Kanten. Eine genauere Untersuchung ist bei der Kleinheit der Krystalle nicht möglich. Das aus Grahams Salz, welches ich nach Tammanns ${ }^{1}$ ) Vorschrift darstellte, gewonnene Strychninsalz zeigt ähnliche Verhältnisse, doch liess sich bei der Kleinheit des Objects nichts Sicheres über die Identität aussagen.

Leider stiess die weitere Untersuchung des Salzes auf Schwierigkeiten, da es sich beim Umkrystallisiren anscheinend theilweise zersetzte. Aus einer "Plasminsäure», die 27\% Phosphor enthielt, gewann ich nach einmaligem Umkrystallisiren ein eisenfreies Präparat, welches bei der Analyse folgende Zahlen ergab:

0,1925 g bei $134^{\circ}$ getrocknet gaben mit Bleichromat gemischt verbrannt $0,4202 \mathrm{~g} \mathrm{CO}_{2}$ und $0,0968 \mathrm{~g} \mathrm{H}_{2} \mathrm{O}$.

$0,2222 \mathrm{~g}$ bei $134^{\circ}$ getrocknet gaben mit Soda und Salpeter verascht nach der Molybdänmethode $0,0562 \mathrm{~g} \mathrm{Mg}_{2} \mathrm{P}_{2} \mathrm{O}_{7}$.

1) C. Tammann, Beiträge zur Kenntniss der Metaphosphate, Journ. f. prakt. Chemie, N. F., Bd. 45, 1892. 


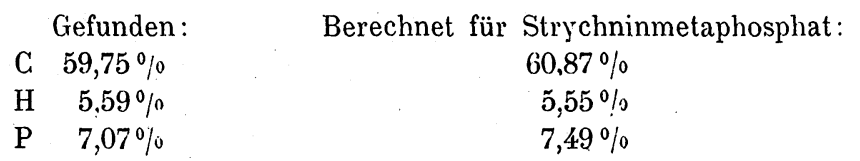

Ein sicherer Schluss auf die Zusammensetzung dieses Salzes kann hiernach noch nicht gezogen werden, doch wird man kaum zweifelhaft sein, dass hier ein Körper vorliegt, der zur Gruppe der Metaphosphorsäuren gehört.

Es ist bemerkenswerth, dass die der Plasminsäure eigenthümliche Phosphorverbindung nicht in gleicher Weise in den Lösungen der Nucleinsäure nachweisbar ist. Es handelt sich bei dieser Metaphosphorsäure um einen eigenartigen Atomcomplex, der neben der "Hefenucleinsäure » in der Hefezelle vorhanden ist und über dessen genetische Beziehungen zur «Hefenucleinsäure» und zum «Nuclein» vorläufig noch nichts bekannt ist.

\section{Ueber das Eisen der Plasminsäure.}

Die nach obiger Methode dargestellte "Plasminsäure» enthält circa $1 \%$ Eisen. Dieses Eisen ist

1. durch Ferrocyankalium und Salzsäure gewöhnlich nicht nachweisbar; doch manchmal tritt. bei Salzsäurezusatz die Reaction ein, ob deshalb, weil die Grenze des fester gebundenen Eisen überschritten ist, muss ich dahingestellt lassen. Aehnliches gilt für die Rhodanprobe.

2. Nach dem Kochen mit Salzsäure tritt die Berlinerblaureaction immer auf. Ebenso verhält sich die Metaphosphorsäure (siehe oben).

3. Es ist als Schwefeleisen nachweisbar, doch tritt die Reaction erst bei Zusatz eines gewissen Ueberschusses an Schwefelammonium zur ammoniakalischen Lösung der Plasminsäure ein; verdünntes Schwefelammonium bleibt entweder ganz wirkungslos oder die Reaction vollzieht sich langsam und es kann eine eingetretene Grünfärbung sogar wieder allmählich verschwinden.

4. Es ist in salzsaurem Alkohol unlöslich; dies geht schon aus der Darstellungsweise hervor, bei der die Substanz 
in einigen Fällen tagelang mit salzsaurem Alkohol in Berührung gestanden und das Eisen doch nicht in Lösung gegangen war.

5. Es wird durch Ammoniak nicht, durch Natronlauge nur allmählich und unvollkommen ausgetällt. Sogar durch direkte Extraction der Plasminsäure mit Natronlauge gelingt es nicht, das Eisen vollkommen zu entfernen.

Man wird durch diese Thatsachen an den Eisengehalt gewisser Nucleinstoffe und Paranucleinstoffe erinnert. Lubavin ${ }^{1}$ ) beobachtete zuerst, dass das aus Milchcasein dargestellte Paranuclein eisenhaltig ist. Bunge ${ }^{2}$ ) stellte unter dem Namen Hämatogen ein eisenhaltiges Paranuclein aus dem Dotter des Hühnereies dar, Zaleski ${ }^{3}$ ) isolirte aus der Leber einen ähnlichen Körper, den er als Hepatin bezeichnete, und Walter ${ }^{4}$ ) gewann ein eisenhaltiges Paranuclein aus dem Dotter des Karpfeneies. Wildenow ${ }^{5}$ ) bestätigte Lubavin's Befund; Hammarsten ${ }^{6}$ ) fand das Pankreasnucleoproteid stark eisenhaltig. Auch Petit ${ }^{7}$ ) isolirte aus Gerste eine hierher gehörige schwefelfreie Substanz, in der das Eisen «à l'état nucleïnique » vorliegt.

Alle diese Präparate waren mit wässeriger oder alkoholischer Salzsäure in Berührung gewesen. Es lag also nahe, eine festere Bindung des Eisens in diesen phosphorhaltigen Stoffen anzunehmen, und Bunge spricht die Ansicht aus, dass

1) Berichte d. deutschen chem. Gesellschaft. X. S. 2237. Ref. von G. Wagner. 1877.

2) Ueber die Assimilation des Eisens. Zeitschr. f. physiol. Chemie, Bd. IX S. $49.188 \check{\text { o. }}$

3) Studien über die Leber. Zeitschr. f. physiol. Chemie, Bd. X, S. 4วิ3. 1886.

4) Zur Kenntniss des Ichtulins und seiner Spaltungsprodukte. Zeitschr. f. physiol. Chemie, Bd. XV, S. 477. 1891.

5) Zur Kenntniss der peptischen Verdauungsprodukte des Caseins. Inaugural-Dissertation. Bern 1893.

6) Zur Kenntniss der Nucleoproteide. Zeitschr. f. physiol. Chemie, Bd. XIX, S. 19. 1894.

7) Sur une nucléine végétale. Comptes Rendus, vol. CXVI, p. 995; 1893. Citirt nach Macallum. 
im Hämatogen eine «organische» Bindung des Eisens vorliege.

Durch meine Beobachtungen über das merkwürdige Verhältniss der Metaphosporsäure zum Eisen veranlasst, stellte ich eine Verbindung von Metaphosphorsäure und Eisen folgendermassen dar. Ich setzte zu Metaphosphorsäure, die ich aus Phosphorsäureanhydrid dargestellt hatte, etwa so viel Eisenchlorid, als durch die überschüssige Säure in Lösung gehalten wurde. Die Lösung wurde mit Ammoniak abgestumpft und durch Alkohol nebst etwas Aether gefällt, mit Alkohol und Aether gut gewaschen, getrocknet und ins Vacuum gebracht. Man erhält so ein weisses, kaum gelbliches Pulver, das in Wasser Salzsäure und Ammoniak löslich ist; letztere Lösung sieht portweinroth aus. Das Eisen ist in der ammoniakalischen Lösung durch wenig Schwefelammonium überhaupt nicht, durch mehr Schwefelammonium mehr oder weniger schnell nachweisbar, je nach der Concentration. Die wässerige Lösung reagirt sauer, gibt mit Silbernitrat einen weissen Niederschlag, wird durch salzsauren Alkohol gefällt. Ich habe diese Substanz tagelang (in einem Versuch vier, im zweiten drei Tage) mit B ung e'scher Flüssigkeit ${ }^{1}$ ) unter häufigem Umschütteln extrahirt und im Filtrate mit Ammoniak und Schwefelammonium kein Eisen nachweisen können; erst wenn das ganze Filtrat eingedampft in ein paar Cubikcentimeter Wasser aufgenommen wurde, war so viel Eisen vorhanden, dass Ammoniak und Schwefelammonium einen schwarzen Niederschlag von Schwefeleisen erzeugten. Es waren also durch den salzsauren Alkohol nur Spuren von Eisen in Lösung gebracht worden, fast das ganze Eisen war im Rückstande zu finden. Aus diesen Thatsachen geht deutlich hervor, dass in dieser Verbindung von Eisen und Metaphosphorsäure das Eisen in einer Form enthalten ist, die sich von der in der Plasminsäure vorliegenden kaum unterscheiden lässt und der Bindung des Eisens in den eisenhaltigen Paranucleinen sehr ähnlich ist.

1) 90 Volumentheile $96 \%$ Alkohols mit 10 Volumen $25 \%$ iger Salzsäure gemischt. 
Nachdem ich nun das Vorhandensein einer zur Gruppe der Metaphosphorsäuren gehörigen Substanz in der "Plasminsäure» nachgewiesen habe, wird man sich der Vermuthung nicht verschliessen können, dass das Eisen auch hier an den in Form von Metaphosphorsäure enthaltenen Phosphor gebunden ist. Es liegt also nahe, in den eisenhaltigen Paranucleinen und Nucleinen eine direkte Bindung des Eisens an Phosphor anzunehmen, speciell in dem Hämatogen, aus welchem nach Altman ${ }^{1}$ ) eine eiweissfällende. Säure hervorgehen soll.

Der Vollständigkeit halber muss ich erwähnen, dass in neuerer Zeit $\mathrm{Macallum}^{2}$ ) das Hämatoxylin als chemisches Reagens zur Unterscheidung des «anorganischen» vom «organischen", an Kohlenstoff gebundenen Eisen empfohlen hat. Marfori ${ }^{3}$ ) hat aber mit demselben Reagens, bei Anwendung einer kleinen Modification, ganz entgegengesetzte Resultate erzielt. Das künstliche Ferratin, welches nach der Macallum'schen Reaction eine "anorgan ische» Eisenverbindung ist, verhält sich der Marfori'schen Modification gegenüber wie eine «organische» Eisenverbindung. Ich selbst habe mit den beiden Reactionen so widersprechende Resultate erzielt, dass ich von einer weiteren Anwendung vorläufig Abstand genommen habe.

Fasse ich nun kurz das Ergebniss meiner Untersuchungen zusammen, so ergibt sich Folgendes:

1. Aus der "Plasminsäure» lässt sich ein krystallisirendes Strychninsalz gewinnen, das sich vom hexametaphosphorsauren Strychnin aus Grahams Salz ${ }^{4}$ ) nicht unterscheiden lässt.

1) Ueber Nucleinsäuren. Archiv für Anatomie und Physiologie. Physiol. Abth. 1889. S. 524 .

2) A new method of distinguishing between organic and inorganic compounds of iron. Journ. of Physiol. 22. S. 92.1897.

3) Sur une nouvelle réaction pour distinguer les composés organiques du fer d'avec les composés anorganiques spécialement par rapport à la ferratine. Arch. ital. de biologie. 30. 1898.

4) Das aus dem Salz Grahams gewonnene Strychninmetaphosphat dürfte ein Gemenge mehrerer Hexametaphosphate sein, da nach Tammann (l. c.) das Salz Grahams ein Gemenge mehrerer (mindestens zweier) Hexametaphosphate ist. 
2. Sowohl die aus der "Plasminsäure» zu gewinnende Phosphorverbindung, wie auch die künstlich dargestellten Metaphosphorsäuren vermögen das Eisen in der Weise zu binden, dass es sich wie "organisches» oder «maskirtes» Eisen verhält.

Vielleicht dürften sich aus dieser bisher unberücksichtigten Bindungsweise des Eisens neue Gesichtspunkte zur Beurtheilung der von $\mathrm{Macallum}^{1}$ ) nachgewiesenen Localisation von Phosphor und Eisen in kernreichen Organen ergeben.

Es ist mir zum Schluss eine angenehme Pflicht, Herrn Prof. A. Kossel für die Ueberlassung des Themas und für sein beständiges Interesse an meiner Arbeit meinen wärmsten Dank auszusprechen.

1) On the distribution of assimilated iron compounds, other than Haemoglobin and Haematin, in animal and vegetable cells. Quarterly Journal of microscopical science. N. S. 38. pag. 175, 1896. - Sowie: On the detection and localisation of phosphorus in animal and vegetable tissues. Proceed. Roy. Soc. Vol. 63, pag. 467. 1898. 